\title{
PEGylated Albumin-Based Polyion Complex Micelles for Protein Delivery
}

Yanyan Jiang, ${ }^{a}$ Hongxu Lu, ${ }^{a}$ Fan Chen, ${ }^{a}$ Manuela Callari, ${ }^{a, c}$ Mohammad Pourgholami, ${ }^{b}$ David L. Morris, ${ }^{b}$ Martina. H. Stenzel ${ }^{* a}$

${ }^{a}$ Centre for Advanced Macromolecular Design, School of Chemistry and School of Chemical Engineering, University of New South Wales UNSW, Kensington NSW 2052, AustraliaEmail: M.Stenzel@unsw.edu.au.

${ }^{b}$ Cancer Research Laboratory, Department of Surgery, St George Hospital, Sydney, NSW 2217, Australia.

${ }^{c}$ Liverpool Hospital Clinical School, and Molecular Medicine Research Group, University of Western Sydney, Sydney NSW 2170, Australia

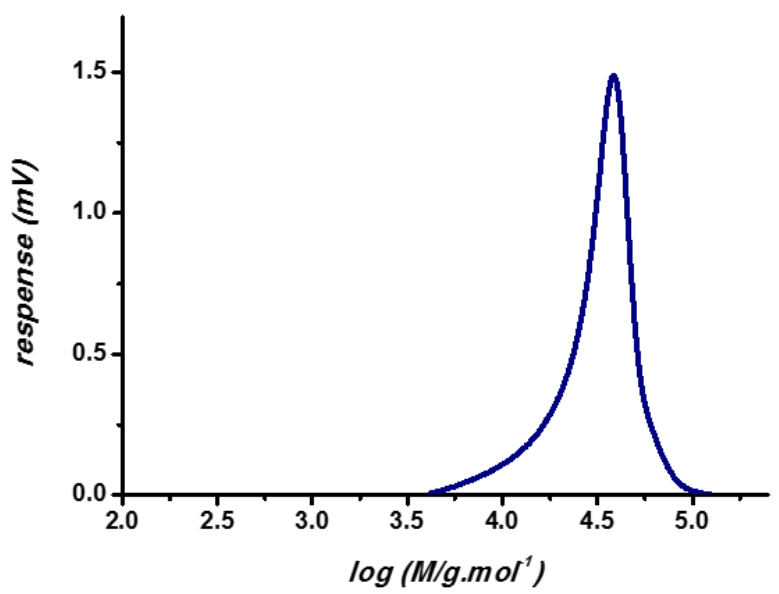

Figure S1 DMAc GPC curve of MI-PEGMEMA. The polydispersity index is 1.2 and the $\mathrm{Mn}, \mathrm{gpc}$ is $31,600 \mathrm{~g} / \mathrm{mol}$. 


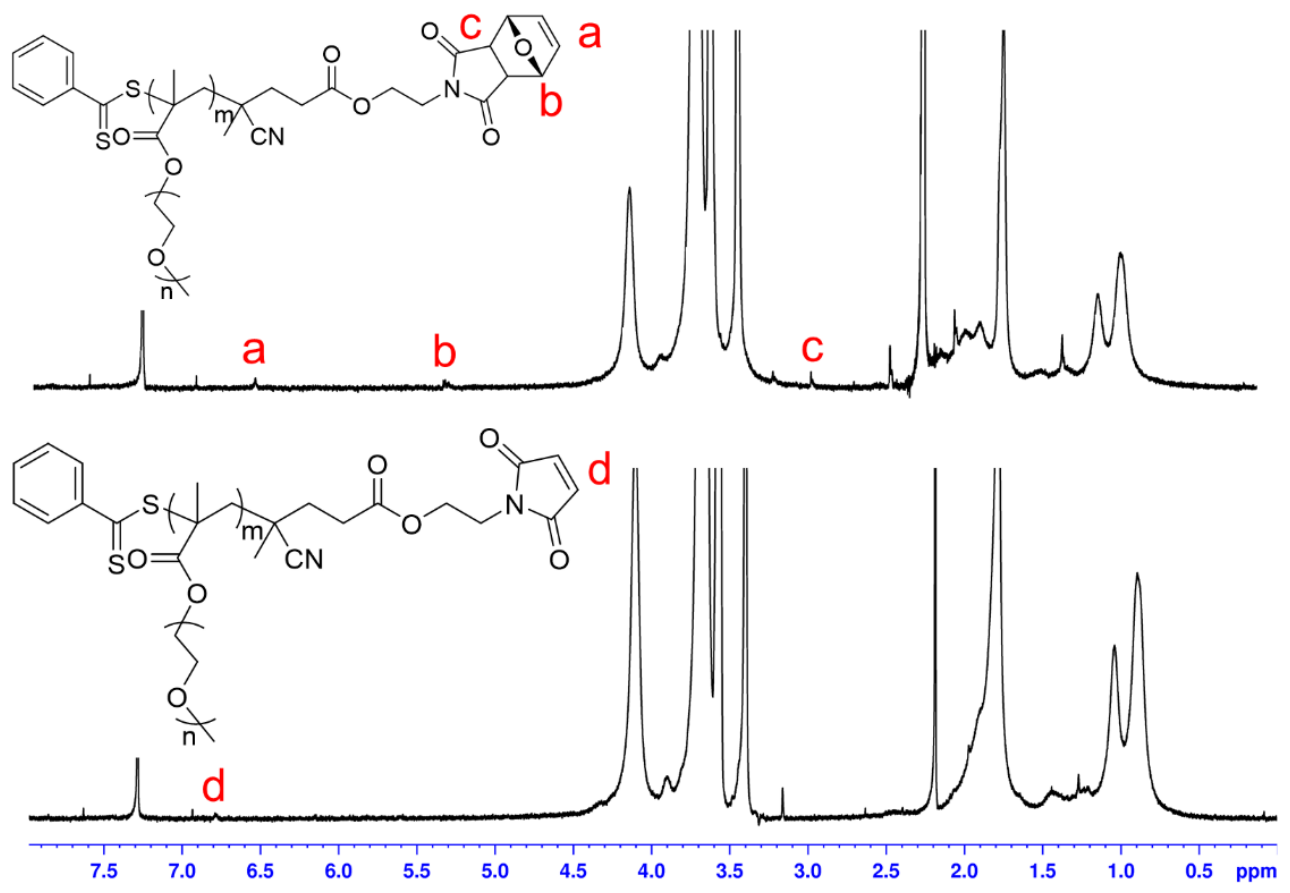

Figure S2 ${ }^{1} \mathrm{H}$ NMR of MI-PEGMEMA in $\mathrm{CDCl}_{3}$ before (top) and after (bottom) deprotection.

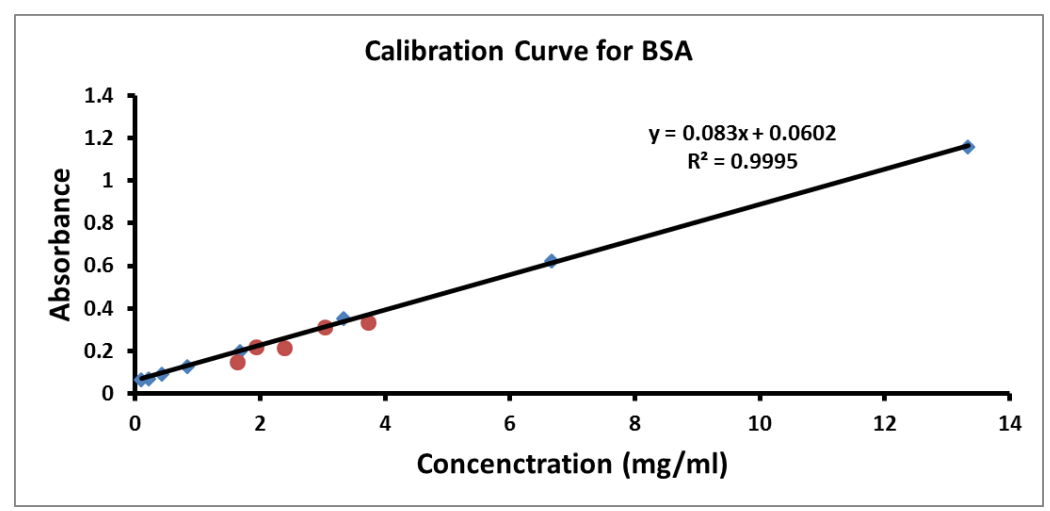

Figure S3 Characterization of PEGMEMA and BSA conjugation using Ellman's assay. The red dots stand for the mixture with different ratios (from left to right, the ratio is 1 to5, 1to2, 1 to 1 to 0.5 and 1 to 0.2 for BSA to PEGMEMA). 
Table S1 Diffusion coefficient of PEGMEMA-BSA

\begin{tabular}{|l|l|}
\hline \multicolumn{2}{|l|}{ PEGMEMA-BSA } \\
\hline-9.0 & -10.08 \\
\hline $1 * 10^{-9}$ & $8.31 * 10^{-11}$ \\
\hline
\end{tabular}

The sample PEGMEMA-BSA shows 2 species: leftover BSA $\left(7.9^{*} 10^{-10}\right)$ and PEGMEMA-BSA $\left(8.31 * 10^{-11}\right)$

(a)

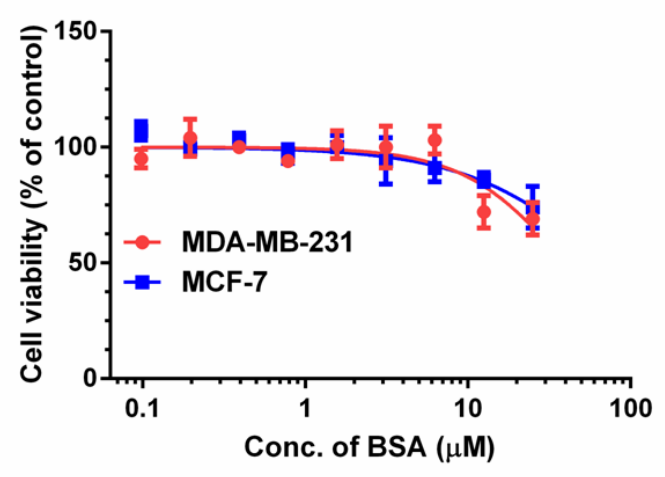

(b) PEG-BSA=1:1

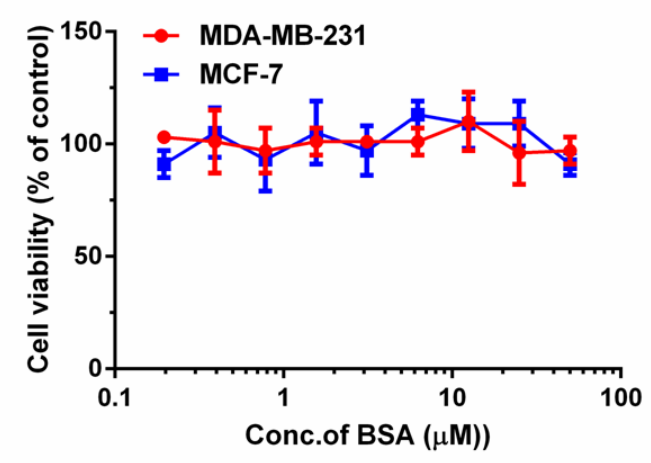

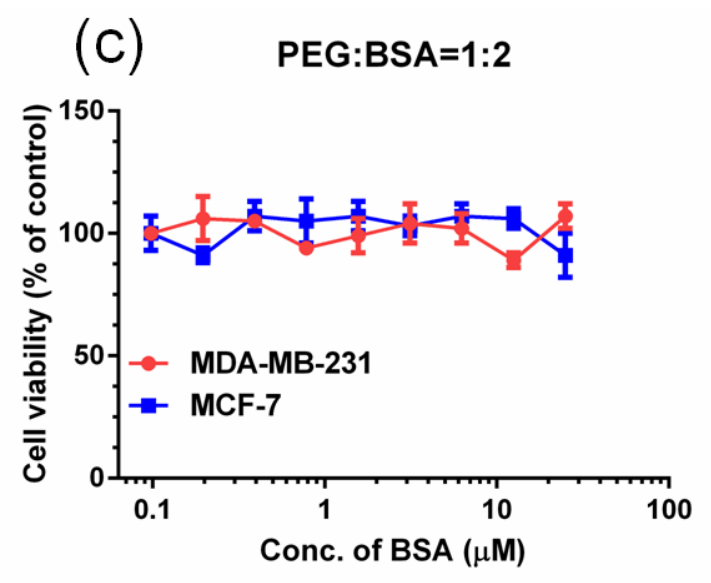

Figure S4 Cytotoxicity assays of PEG-BSA conjugates with different ratios against breast carcinoma cell lines (MDA-MB-231 and MCF-7) for $48 \mathrm{~h}$, and the mean \pm standard deviations are shown. 
Table S2 Size distribution of the BSA/Lyz and PEGMEMA-BSA/Lyz polyplex with different BSA to Lyz ratios.

\begin{tabular}{|c|c|c|c|}
\hline Lyz to BSA & PdI & Lyz to PEGMEMA-BSA & PdI \\
\hline 10 & 0.48 & 10 & 0.28 \\
\hline 5 & 0.36 & 5 & 0.43 \\
\hline 2.5 & 0.38 & 2.5 & 0.31 \\
\hline 1 & 0.81 & 1 & 0.91 \\
\hline 0.5 & 0.33 & 0.5 & 0.65 \\
\hline 0.25 & 0.41 & 0.25 & 0.59 \\
\hline 0.125 & 0.4 & 0.125 & 0.61 \\
\hline
\end{tabular}

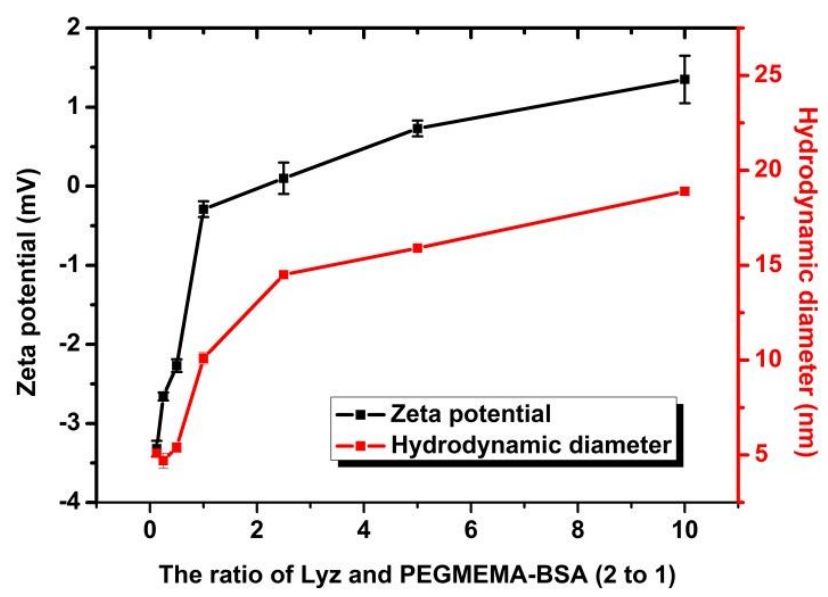

Figure S5. Hydrodynamic diameter and zeta potential variation of PEGMEMA-BSA/Lyz with the increase of lysozyme. The ratio of PEGMEMA and BSA was 2 to 1 . The concentration of BSA in each sample was $1.5 \mathrm{mg} / \mathrm{mL}$.

Table S3 The physical properties of the FITC labeled PEGMEMA-BSA/Lyz micelles.

\begin{tabular}{|l|l|l|l|}
\hline Sample name & $\begin{array}{l}\text { Number } \\
\text { Mean } \\
\text { (d.nm) }\end{array}$ & $\begin{array}{l}\text { Zeta } \\
\text { potential }\end{array}$ & PdI \\
\hline FITC labeled PIC & 27.7 & 4.95 & 0.35 \\
\hline
\end{tabular}




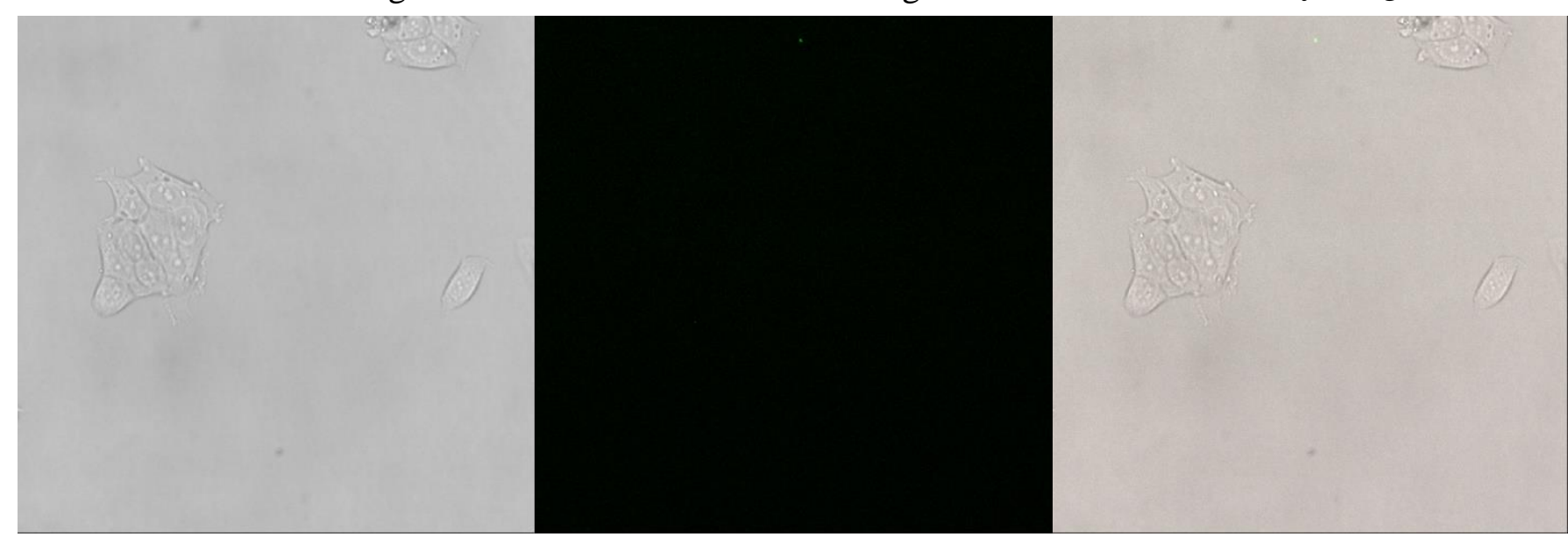

Figure S6. Free FITC-labelled lysozyme in MCF-7 after 2 hours incubation. 